\section{0 conceito de perspectiva na rede conceptual das construções completivas epistêmicas: o caso das construções intersubjetivas disjuntivas}

The concept of perspective within the conceptual network of epistemic complementation clauses: the case of intersubjective disjunctive constructions

Sandra Aparecida Faria de ALMEIDA (UFJF) sandra.sf@gmail.com

Recebido em: 31 de jan. de 2019. Aceito em: 05 de jun. de 2019.
ALMEIDA, Sandra Aparecida Faria de. O conceito de perspectiva na rede conceptual das construções completivas epistêmicas: o caso das construções intersubjetivas disjuntivas. Entrepalavras, Fortaleza,

Resumo: Este artigo tem como objetivo discutir a noção de (inter) subjetividade com base na Gramática Cognitiva de Langacker $(1987,1990,2008)$, em especial no tocante à noção de perspectiva, e em outros autores que tratam do tema. Compreende-se o conceito de intersubjetividade como um mecanismo que estabelece um elo conceptual entre as construções completivas epistêmicas em inglês, em especial aquelas de moldura sintática do tipo [SN VEpist [that S]], que tendem a co-ocorrer com outras construções intersubjetivas complexas, como as construções pseudo-clivadas, as construções condicionais, as construções interrogativas e as construções negativas. A partir de dados reais de fala coletados por Almeida (2010) bem como retirados de corpora eletrônicos como o BNC e o COCA, postula-se que o conceito de intersubjetividade se verifica tanto no plano linguístico, em termos explícitos à estrutura linguística, demonstrando atenção à perspectiva oferecida pelo interlocutor (TRAUGOTT; DASHER, 2005); quanto em termos implícitos, na atitude do falante ao conceptualizar o evento de fala sob uma determinada perspectiva (LANGACKER, 1987, 1990, 2008; VERHAGEN, 2005; NUYTS, 2001), 
que não se encontra alinhada com as demais disponíveis no fluxo discursivo. Os dados analisados confirmam a hipótese de que as construções sintáticas em relevo exibem intersubjetividade tanto em termos conceptuais quanto estruturais, permitindo reivindicar o conceito como um elo conceptual que une essas construções em rede.

Palavras-chave: Construções completivas epistêmicas. (Inter)subjetividade. Perspectiva.

Abstract: This work is aimed at discussing the notion of (inter) subjectivity according to Langacker's $(1987,1990,2008)$ Cognitive Grammar as well as to other authors who deal with the subject. The concept is understood as a conceptual device for linking epistemic complex sentences in English, herein the ones represented by the [SN VEpist [that S]] structure, which co-occur with other intersubjective constructions, such as pseudo-clefts, conditional clauses, questions and negative sentences in a cognitive disjunction procedure. With basis on real corpus data from Almeida (2010), made of 382 occurrences of both [SN VEpist [that S]] and [SN VEpist [ $\varnothing$ S]] argument structures, as well as from BNC and COCA online corpora, intersubjectivity is viewed as a surface mechanism of attention to the addressee (TRAUGOTT; DASHER, 2005) as well as an implicit device for conceptualizing the speaker's point of view (LANGACKER, 1987, 1990, 2008; VERHAGEN, 2005; NUYTS, 2001) as distinct from the others activated in discourse, thus conveying a non-alignment procedure towards the perspective available in the discourse flow. Data showed that such constructions convey intersubjectivity both in explicit and implicit terms, thus allowing the notion to be viewed as a device linking such constructions in a conceptual network.

Keywords: Epistemic Complex Clauses. (Inter) subjectivity. Perspective.

\section{Introdução}

O conceito de (inter) subjetividade tem sido objeto de estudo de diferentes pesquisadores, tanto no que tange à cognição humana em geral, quanto no que se refere ao uso da linguagem (cf. LANGACKER, 1987, 1990, 2008; TRAUGOTT; DASHER, 2005; VERHAGEN, 2005; NUYTS, 2001).

Em termos de linguagem, alguns autores compreendem o fenômeno como sendo algo codificado na expressão linguística e, portanto, explícito nos enunciados produzidos (cf. TRAUGOTT; DASHER, 2005). Outros autores, porém, concebem a (inter)subjetividade como algo implícito à forma linguística e que só pode ser compreendido em termos discursivo-pragmáticos (cf. LANGACKER, 1987, 1990, 2008; VERHAGEN, 2005; NUYTS, 2001).

A partir de uma revisão dos conceitos de objetividade, subjetividade e intersubjetividade na literatura linguística contemporânea (cf. LANGACKER, 1987, 1990, 2008; TRAUGOTT; DASHER, 2005; VERHAGEN, 2005; e NUYTS, 2001), este artigo ${ }^{1}$ visa a

${ }^{1} \mathrm{O}$ tema deste artigo é um desmembramento da tese de doutorado do autor (ALMEIDA, 2010). 
V. 9 (2)

$214-236$

maio-ago

2019

contribuir para o aprofundamento do conceito de (inter) subjetividade como um mecanismo capaz de estabelecer elos construcionais (GOLDBERG, 1995, 2006) entre as estruturas linguísticas completivas, evidenciando processos de não-alinhamento de perspectivas discursivas, a que se denomina mecanismos de disjunção cognitiva. ${ }^{2}$

Tal procedimento, argumenta-se, pode ser observado no uso de construções completivas epistêmicas na língua inglesa do tipo [SN VEpist [that S]], como em "But certainly I don't think that many people are overtaxed." ("Mas, certamente, não acho que muitas pessoas estão sobrecarregadas com impostos"), que co-ocorrem com construções pseudo-clivadas, construções condicionais, construções interrogativas e construções negativas. Postula-se que tais construções indicam (inter) subjetividade de maneira explicita, através de operadores discursivos sinalizadores de atenção à perspectiva do interlocutor e codificados na forma linguística, bem como de maneira implícita, na atitude do falante com relação à perspectiva ativada no fluxo discursivo, em um mecanismo de não alinhamento de perspectivas. Dessa forma, o estudo busca sintetizar ambas as visões dos pesquisadores no que tange à (inter) subjetividade.

A presente análise se baseia no corpus coletado por Almeida (2010), com dados de textos jornalísticos, extraídos virtualmente de fontes, tais como a CNN, BBC, $\mathrm{ABC}$ etc., de revistas especializadas em artigos em inglês, como a Speak Up e a Newsweek, além de blogs e fóruns de discussão. A amostra contém um total de 382 ocorrências de construções completivas epistêmicas dos tipos [SN VEpist [that S]] e [SN VEpist $[\varnothing \mathrm{S}]]$ e tem um viés qualitativo, colocando em relevo, como foi dito anteriormente, as construções sintáticas que co-ocorreram com a construção completiva epistêmica em que o complementizador é utilizado, isto é, as do tipo [SN VEpist [that S]],tendo o preenchimento do slot verbal com o verbo think, que se mostrou o mais frequente da amostra (cf. ALMEIDA, 2010, ALMEIDA; FERRARI, 2012).

\section{(Inter) subjetividade}

A noção de (inter) subjetividade tem sido abordada nos estudos sobre diferentes aspectos da cognição humana e, no que tange

\footnotetext{
${ }^{2}$ Este artigo trata apenas dos casos de não-alinhamento de perspectivas. Os casos, igualmente intersubjetivos, classificados como sinalizadores de conjunção cognitiva (ALMEIDA, 2010; ALMEIDA; FERRARI, 2012), isto é, que expressam alinhamento entre perspectiva ativada e perspectiva assumida, estão fora do escopo deste trabalho.
} 
à linguagem, o conceito tem ganhado ainda mais proeminência (cf. LANGACKER, 1987, 1990, 2008; TRAUGOTT; DASHER, 2005; VERHAGEN, 2005;NUYTS, 2001). Embora alguns desses autores enfoquem a noção sob o prisma linguístico, identificando as marcas da intersubjetividade explicitamente na forma linguística, outros pesquisadores assumem uma postura essencialmente discursivo-pragmática e a concebem como um recurso implícito à gramática da língua, que somente pode ser interpretado discursivamente.

Traugott \& Dasher (2005), por exemplo, se alinham com a primeira abordagem, visto que para eles a subjetividade é codificada na língua pelo uso da dêixis, da modalidade e de marcadores discursivos, por exemplo. Para os autores, a sentença "I will take you to school" ("Vou levá-lo para a escola") expressa subjetividade através dos pronomes "I" e "you", que deiticamente fazem alusão ao falante e ouvinte. A intersubjetividade, por sua vez, implica a atenção do falante com relação ao seu interlocutor no evento de fala. O falante, dessa forma, sinaliza a "inclusão" do ouvinte na sua expressão linguística enquanto participante do evento comunicativo. Essa sinalização pode ser expressa por pronomes pessoais, dêiticos e vários outros recursos. Para ilustrar, Traugott e Dasher se utilizam do mesmo exemplo acima, acrescido da palavra "actually", que funciona como um hedge ou marcador discursivo: "Actually, I will take you to school." ("Na verdade, vou levá-lo para a escola."). Essa sentença assume valor intersubjetivo na medida em que funciona pragmaticamente como mecanismo de atenção do falante ao seu interlocutor (em resposta a algo dito ou perguntado por este). Nas palavras de Traugott e Dasher (2005, p. 22), "a subjetividade é um pré-requisito para a intersubjetividade na medida em que a atitude do falante/escritor com relação ao ouvinte/leitor é uma função da perspectiva do falante/escritor”.

Por outro lado, para Langacker (1990), a conceptualização de um evento comunicativo é a forma como se representa uma determinada cena, e a organização, a disposição ou o "arranjo" dessa cena é a perspectiva assumida pelo falante para representá-la. Para ele, os conceitos de subjetividade e objetividade estão intimamente relacionados ao conceito de ponto de vantagem (vantage point), que representa a localização real ou imaginária (projeção) do falante face à cena comunicativa. Em outras palavras, esse conceito diz respeito ao ponto ou a partir de que perspectiva o falante conceptualiza a cena. Se falante e ouvinte se utilizarem de pontos distintos, suas 
V. 9 (2)

$214-236$

maio-ago 2019 perspectivas serão também distintas. Embora falante e ouvinte sejam considerados conceptualizadores ou viewers e, portanto, sujeitos da conceptualização, nem sempre eles "figuram" explicitamente nas expressões linguísticas produzidas na cena comunicativa. Sua presença está, assim, implícita. Para ilustrar essa diferença, Langacker se utiliza dos seguintes exemplos:

(1) Vanessa jumped across the table.

(2) The new highway goes/runs/climbs from the valley floor to the senator's mountain lodge.

No primeiro caso, a construção é tomada como maximamente objetiva, pois o conceptualizador observa a cena e não é parte dela. No segundo caso, o conceptualizador se projeta na cena, através de um percurso mental e não físico, tornando a construção maximamente subjetiva.

Para Verhagen (2005), a habilidade de perceber a si próprio e os outros como agentes intencionais caracteriza o conceito de subjetividade. Esse conceito está atrelado ao de argumentatividade, como sendo a capacidade que as pessoas têm em se "coordenar" cognitivamente umas com as outras a partir do uso que fazem da língua na interação comunicativa, ou seja, no fluxo discursivo, para induzir ou realizar inferências e criar um discurso coerente. Assim, para Verhagen, subjetividade implica (i) a conceptualização de um objeto por parte de um sujeito, incorporando a ideia de que os pensamentos e as crenças desse falante podem diferir do mundo real (o que se opõe ao conceito de objetividade); (ii) um ponto de vista por parte do falante que é distinto dos demais, no sentido de ser pessoal e não compartilhado, como no caso de crenças e opiniões. É esse segundo aspecto que Verhagen denomina intersubjetividade: a coordenação mútua ou comunicação de sistemas cognitivos. Em outras palavras, falante e interlocutor interagem com base em seus respectivos sistemas cognitivos que se encontram alinhados.

Para ilustrar os conceitos de subjetividade e objetividade, Verhagen (2005) se utiliza dos seguintes exemplos:

(3) The debate promises to be exciting.

(4) He promises to defend the constitution. 
No primeiro caso, a construção é tida como subjetiva, na medida em que se situa no nível da intersubjetividade, isto é, envolve os sujeitos da conceptualização, falante e ouvinte, embora não explicitamente expressos na sentença, ou seja, na expressão linguística, em uma perspectiva avaliativa do evento. No segundo caso, a construção é considerada como objetiva, pois focaliza um aspecto do objeto da conceptualização, a defesa da constituição (elemento focalizado) durante o debate (objeto da conceptualização).

Nuyts (2001), por sua vez, entende a subjetividade como estando relacionada à maior ou menor evidência de que o falante dispõe para realizar seu julgamento epistêmico. O autor reanalisa os conceitos de subjetividade/objetividade a partir de uma polaridade subjetividade/ não-subjetividade, que evolui para a díade subjetividade/intersubjetividade. Nuyts argumenta que a diferença entre esses dois pólos está no domínio da evidencialidade e não no domínio da epistemicidade. Em suas palavras, a subjetividade "envolve a indicação por parte do falante de que só ele sabe (ou tem acesso) à evidência e tira conclusões a partir dela" (NUYTS, 2001, p. 393), enquanto a intersubjetividade "envolve a indicação por parte do falante de que a evidência é conhecida (ou pode ser acessada) por um grupo maior de pessoas que compartilham a mesma conclusão baseada nesta evidência." (NUYTS, 2001, p. 393) Para ilustrar sua abordagem, vejamos os exemplos a seguir:

(5) ...but I think that also in believing there are ups and downs, because to me believing has everything to do with life and vice-versa.

(6) Now that I contradict very fundamentally. You could organize a thousand meetings with those who are against it. You wouldn't at all change their saying "not here". I think you have to see that.

(7) I think now I have to say something after all worthy colleague.

Para Nuyts, o exemplo (5) caracteriza subjetividade, pois expressa experiência pessoal, cuja evidência está disponível apenas para o falante e não é compartilhada pelas pessoas. No exemplo (6) a visão do falante está em oposição à do seu interlocutor, sinalizando, assim, intersubjetividade. Já o exemplo (7) mostra o uso do verbo epistêmico como "atenuador" ou "hedge" discursivo que, mais uma vez, se constitui em um aspecto da intersubjetividade, na medida em que envolve atenção ao ouvinte. 
V. 9 (2)

$214-236$
Em síntese, embora esses autores atrelem a subjetividade e a intersubjetividade a uma questão de ponto de vantagem ou perspectiva, ou de argumentatividade, ou de evidencialidade ou mesmo a um mecanismo de coordenação cognitiva, todos parecem concordar que, no caso das construções epistêmicas, a oração principal desempenha o papel de expressar a forma como o falante conceptualiza o conteúdo expresso na oração encaixada, e que a relação entre essas orações, mais que sintática, é conceptual. Da mesma forma, a atitude que o falante assume sobre esse conteúdo se traduz discursivo-pragmaticamente de forma explícita na estrutura linguística, através do preenchimento do slot verbal com verbos epistêmicos e dos operadores de (inter)subjetividade, bem como de forma implícita, através de mecanismos de alinhamento ou não alinhamento de perspectivas, como veremos a seguir.

No Brasil, Almeida (2010) e Almeida e Ferrari (2012) delinearam uma noção de (inter)subjetividade que busca integrar as contribuições desses diferentes autores já que, a seu ver, os conceitos de certa forma se sobrepõem e se complementam em alguns aspectos. Para as autoras, as noções de subjetividade e intersubjetividade servem ao propósito de indicar a codificação linguística do "ground" (o evento de fala, seus participantes e as circunstâncias imediatas da interação, como tempo e espaço), tanto em termos explícitos (como apontam Traugott e Dasher (2005)), como em termos implícitos (como defendem Langacker (1990) e Verhagen (2005)). Dessa forma, construções do tipo I think that/ $\varnothing$ it's interesting podem ser consideradas mais subjetivas, e suas contrapartes formadas por orações independentes mais objetivas (It's interesting). As primeiras perfilam elementos do "ground" (em geral, falante/ouvinte), enquanto as últimas mantêm a referência ao falante/ouvinte fora do escopo da predicação. Por outro lado, as primeiras podem também ser consideradas mais intersubjetivas, já que, na visão dessas autoras, elas implicitamente sinalizam um alinhamento ou não alinhamento com perspectivas ativadas discursivamente sobre um determinado evento de fala, conforme veremos mais adiante.

Almeida (2010) e Almeida e Ferrari (2012) argumentam também que o preenchimento do slot verbal das construções [SN VEpist [that S]] e [SN VEpist [ $\varnothing \mathrm{S}]$ ] em inglês pode ocorrer com diferentes tipos de verbos epistêmicos, como guess, suppose, think, believe, find e know, fazendo, assim, referência ao "ground" por vias diferenciadas. As construções com os verbos "to suppose (that)" e "to guess (that)" são consideradas sinalizadoras de referência ao "ground" via uma suposição 
perfilada e inerente à proposição, que o falante compartilha ou não com o ouvinte e outros. Construções com o verbo "to think (that)" se relacionam ao "ground" via o perfilamento de uma determinada opinião que faz parte do universo conceptual do falante e que pode ou não ser compartilhada pelo interlocutor e outrem. Já as construções com o verbo "to believe (that)" são tidas como sinalizadoras de referência ao "ground" via o perfilamento de uma determinada crença inerente ao domínio conceptual do falante. As construções com o verbo "to find (that)", por sua vez, se relacionam ao perfilamento de uma determinada experiência por parte do falante que pode ser compartilhada ou não pelo interlocutor e outrem, mas que se apoia na realidade. Por fim, as construções com o verbo "to know (that)" são tidas como sinalizadoras do perfilamento de uma informação socialmente compartilhada, que, dessa forma, pertence ao universo conceptual do falante bem como do ouvinte e de terceiros.

Em termos de assertividade, as autoras postulam ainda a existência de um continuum entre esses verbos, visto que verbos como suppose, guess e think exibem menor assertividade e demandam menor força ilocucionária, sendo, portanto, compatíveis com contextos de alinhamento de perspectivas. Verbos como believe, find e know, porém, são tidos com mais assertivos e tendem a ocorrer em contextos que exigem maior força ilocucionária por parte do falante, como nos casos em que não há alinhamento entre as perspectivas ativadas discursivamente.

\section{As construções completivas epistêmicas}

Na visão de Palmer (1986), muitas das orações subordinadas, principalmente aquelas que funcionam como objeto direto, como é o caso das orações completivas epistêmicas, "reportam as atitudes e opiniões dos sujeitos das orações principais, que são apresentados como os falantes que expressaram, ou podem ser considerados como tendo expresso, algum tipo de modalidade" (PALMER, 1986, p.126). Vejamos alguns exemplos:

(8) He concluded that she was there.

(9) He urged her to be there.

Nos exemplos acima, a oração principal exibe um processo dedutivo sobre o conteúdo da oração subordinada no primeiro caso e um pedido no segundo, isto é, a função da construção epistêmica pode 
V. 9 (2)

$214-236$

maio-ago

2019

se verificar no plano epistêmico ou performativo. Em outras palavras, o conteúdo da oração principal, através da seleção de seu verbo epistêmico, indicará a natureza desse processo.

Tomasello $(2003,2005)$, por sua vez, observa que a criança tende a adquirir primeiro as construções que expressam atitudes psicológicas que sinalizam (i) intenção, desejo, compulsão (querer V, tentar V, ter de V) e (ii) percepção, crença (achar que $\mathrm{P}$, saber que $\mathrm{P}$, ver se P). Em segundo lugar, vêm as construções identificacionais do tipo "That's the doggie that barks all the time" ("Esse é o cachorrinho que late o tempo todo") e em terceiro lugar, estão as orações com menor ligação entre si, do tipo "If $X$ then $Y$ " ou "X because of $Y$ ". No caso específico das construções com complementos sentenciais, Tomasello observa que os verbos da oração matriz, tais como "to think, to know, to believe e to say" se assemelham aos verbos modais epistêmicos no sentido de que expressam certeza, percepção, conhecimento e são usados em expressões curtas e formulaicas do tipo "I thinkP" ou "I know P" que são sempre na primeira pessoa, no presente simples, na forma afirmativa, com o intuito de expressar uma atitude quanto ao conteúdo da outra oração ou chamar a atenção do ouvinte sobre esse conteúdo.

Dentro dessa mesma concepção, Langacker (2008) argumenta que as construções complexas, tradicionalmente denominadas de "subordinadas", contêm uma proposição "embutida" na oração principal ou matriz, o que faz da oração encaixada, segundo a terminologia tradicional, um constituinte interno da oração matriz. No entanto, como observa esse autor, a relação de subordinação deve ser entendida do ponto de vista conceptual, no sentido de que a oração erroneamente chamada de "principal" nada mais é do que a expressão de uma configuração mental sobre o conteúdo expresso na oração dita "subordinada", isto é, a oração matriz expressa a perspectiva sob a qual a proposição contida na oração subordinada vai ser expressa.

Em consonância ao pensamento de Langacker, Verhagen (2005) postula que a oração principal funciona como um mecanismo linguístico de que o falante dispõe para "convidar o interlocutor a assumir uma determinada perspectiva sobre um objeto de conceptualização que está representado na oração encaixada" (p.79). Assim, na sua visão, a oração matriz tem o papel de indicar uma relação intersubjetiva entre falante e interlocutor, e não uma relação estritamente objetiva entre falante e objeto descrito. Nesse aspecto, como argumenta Verhagen, as construções completivas podem ser 
concebidas como um construto linguístico capaz de sinalizar operações cognitivas entre sujeitos, corroborando o argumento de Goldberg (1995, 2006) em favor de construções que estabelecem entre si uma estrita relação entre forma e significado e que são passíveis de serem categorizadas como pertencentes a uma mesma rede de significação. O verbo da oração matriz, segundo Verhagen (2005), "funciona como um operador sobre a relação entre os conceptualizadores, permitindo ao falante/escritor sugerir vários graus de identificação com a perspectiva que é "colocada em cena" (p. 80). Assim, enunciados do tipo "X thinks/ promises/hopes that $Y$ " representam um determinado evento como objeto da conceptualização, mas podem ser tomados como uma instrução por parte do falante/escritor para que o ouvinte/leitor conceptualize Y de uma determinada forma.

No Brasil, pesquisas anteriores (cf. ALMEIDA, 2010; ALMEIDA; FERRARI, 2012) abordam a questão da (inter)subjetividade no uso das construções completivas epistêmicas a partir da moldura teórica da Gramática de Construções (GOLDBERG, 1995, 2006), em especial com relação ao Princípio da Não-Sinonímia, segundo o qual "se duas construções são sintaticamente distintas, tais construções devem ser também distintas semântica ou pragmaticamente." (GOLDBERG, 1995, p.67). Desse princípio, Goldberg extrai o seguinte corolário: "se duas construções são sintaticamente distintas e semanticamente sinônimas, então elas não podem ser pragmaticamente sinônimas." (GOLDBERG, 1995, p.67).

Ao analisarem as construções completivas epistêmicas do tipo [SN VEpist [that S]] e [SN VEpist [ $\varnothing$ S]] em inglês, construções essas que são sintaticamente distintas mas semanticamente sinônimas, Almeida (2010) e Almeida e Ferrari (2012) postularam que elas também são pragmaticamente diferentes entre si, na medida em que atendem a objetivos discursivo-pragmáticos distintos. As autoras argumentam que as construções do tipo [SN VEpist [that S]] tendem a exibir um comportamento discursivo-pragmático de não-alinhamento de perspectivas: aquela que o falante assume para si e a que está ativada no fluxo discursivo, seja pelo interlocutor, pelo próprio falante em um momento anterior ou porque é socialmente compartilhada. A esse tipo de comportamento denominou-se disjunção cognitiva e, dessa forma, essas construções são tratadas no presente artigo como construções intersubjetivas disjuntivas. O exemplo a seguir ilustra esse tipo de uso: 
V. 9 (2)

$214-236$

maio-ago

2019

(10) Josef Xercavins: If we talk about the UN reform, we are producing some feeling of weakness of UN, and I think that our goal should be to strengthen the UN. And the best reform of the UN that we can do is to put the...all of the international institutions under the umbrella of the UN. This is, of course, not in opposition of the ideas of the secretary general; of course it's not. But I think that our best way to help him is to say that the best reform of UN is to put the World Bank, the World Trade Organization in the hands of UN with our participation. (SPEAK UP $n^{\circ}$ 194, 2003, p.24-25)

No caso acima, o falante se utiliza de uma perspectiva presumida, e que é socialmente compartilhada, a de que as Nações Unidas estão enfraquecidas, para expor seu ponto de vista, que é contrário a essa perspectiva: na opinião do falante, as Nações Unidas deveriam ser fortalecidas.

Em se tratando da construção [SN VEpist [ $\varnothing \mathrm{S}]$ ], as autoras postularam um comportamento discursivo-pragmático distinto. Para elas, a construção sinaliza um alinhamento de perspectivas: a que se encontra ativada discursivamente, seja pelo falante, seja pelo interlocutor, seja por um conhecimento presumido ou compartilhado e a que o falante endossa em sua interação verbal. O exemplo abaixo exibe esse tipo de ocorrência:

(11) A:Which of the following will weigh more heavily in the election: the economy or Iraq?"

B: "I think for most Americans the economy will weigh more heavily, because the majority of people are most concerned with that which most directly affects their lives - having good jobs, enough food on the table, enough money to care for their families." (SPEAK UP no 209, 2004, p. 14-15)

No caso acima, há duas perspectivas ativadas na pergunta do entrevistador: (i) a economia irá pesar nas próximas eleições; (ii) a guerra do Iraque irá pesar nas próximas eleições. O entrevistado se alinha com uma delas. A esse tipo de comportamento denominou-se conjunção cognitiva. 
Outro aspecto discursivo-pragmático relevante nos dados observados em pesquisas anteriores (ALMEIDA, 2010; ALMEIDA; FERRARI, 2012) e retomados para essa pesquisa foi a presença de operadores de intersubjetividade, isto é, estruturas sintáticas específicas, tais como as construções pseudo-clivadas, as construções condicionais, as construções interrogativas e as construções negativas, além de marcadores discursivos de intersubjetividade, conforme categorização de Traugott e Dasher (2005), que tendem a co-ocorrer com a construção epistêmica com o complementizador ([SN VEpist [that S]]). Essa tendência parece estar associada ao fato de que esses recursos sintáticos já carregam alguma carga de intersubjetividade, visto que são estruturas de ênfase e/ou foco na perspectiva que o falante deseja reivindicar sobre o assunto, mas em atenção a outra perspectiva explícita ou presumida na interação, da qual o falante diverge ou faz oposição, em um procedimento de disjunção cognitiva.

Ainda segundo os princípios da organização linguística propostos por Goldberg $(1995,2006)$, as construções podem estabelecer entre si elos ou laços de polissemia, elos ou laços de subparte, elos ou laços de instanciação e elos ou laços de extensão metafórica (GOLDBERG, 1995, p.75-81). Com base no conjunto de construções objeto desse estudo, denominadas construções completivas epistêmicas intersubjetivas disjuntivas, defende-se que a relação construcional se dá com base em laços de instanciação, isto é, "uma dada construção é uma instância de outra construção se e somente se uma construção é uma versão mais específica da outra" (GOLDBERG, 1995, p. 79). Assim, argumenta-se que a construção completiva epistêmica pode existir independentemente de quaisquer de suas versões: a pseudo-clivada, a negativa, a interrogativa ou mesmo a condicional. Essas, porém, exibem (inter) subjetividade em um duplo procedimento de disjunção cognitiva (daí o nome de construções disjuntivas), pois sinalizam implícita e explicitamente o não alinhamento de perspectivas no fluxo discursivo.

Finalmente, considerando os quatro tipos de construção que co-ocorrem com a construção completiva epistêmica, argumenta-se que os quatro tipos de construção coincidem com os tipos de sentenças que carregam uma pressuposição em nível pragmático, que pode ser recuperada linguisticamente em termos sintáticos e semânticos (cf. CANÇADO, 2012). Em outras palavras, segundo a autora, a estrutura lingüística oferece elementos ou pistas para que se compreenda um conteúdo pressuposto (p.37). Aplicando esse viés semântico-pragmático ao conjunto de construções 
V. 9 (2)

$214-236$

maio-ago 2019 objeto desse estudo, temos que a pseudo-clivagem, a negação, a interrogação e a condicionalidade não anulam ou cancelam o conteúdo pressuposto da construção completiva epistêmica, mas o coloca em proeminência ou em perspectiva, atribuindo a ele força ilocucionária.

Passaremos agora à metodologia e procedimentos de análise para esses quatro tipos de construções.

\section{Metodologia}

Para o presente artigo, serão colocadas em relevo as construções completivas epistêmicas do tipo [SN VEpist [that S]], que ocorrem na presença de outras construções complexas, como as construções pseudoclivadas, condicionais, interrogativas e negativas, que denominamos coletivamente de construções completivas epistêmicas intersubjetivas disjuntivas. Dessa forma, postula-se nesse artigo que:

(i) o conceito de intersubjetividade se constitui em um elo construcional entre as construções complexas que coocorrem com a construção [SN VEpist [that S]], formando redes conceptuais;

(ii) essas construções completivas epistêmicas indicam (inter) subjetividade de maneira explícita, através de operadores discursivos sinalizadores de atenção à perspectiva do interlocutor e codificados na forma linguística, bem como de maneira implícita, na atitude do falante com relação à perspectiva ativada no fluxo discursivo, em um mecanismo de disjunção cognitiva.

Para efeitos de análise, utilizaremos a base de dados retirada de Almeida (2010), composta de ocorrências das construções dos tipos [SN VEpist [that S]] e [SN VEpist [ $\varnothing \mathrm{S}]$ ] em inglês, que tiveram o preenchimento do slot verbal na oração principal por diferentes tipos de verbos epistêmicos, como guess, suppose, think, believe, find e know, bem como ocorrências de corpora de língua inglesa, como o British National Corpus (BNC) e o Corpus of Contemporary American English (COCA), restritas aos quatro tipos de construção em análise no presente artigo.

Neste trabalho, serão discutidos os casos com preenchimento do slot verbal com o verbo "think" que em estudos anteriores (cf. ALMEIDA, 2010; ALMEIDA; FERRARI, 2012) se mostrou mais frequente. Realizaremos, 
então, uma análise qualitativa de cada uma das construções que coocorrem com a construção epistêmica complexa, doravante CEC, e procuraremos estabelecer algumas considerações face ao conceito de intersubjetividade.

\section{As construções pseudo-clivadas}

As construções pseudo-clivadas, como observam Quirk e Greenbaum (1973) são aquelas construções enfáticas iniciadas com o pronome "what", como em "What I'd really like is a nice hot bath." Segundo esses autores, a construção pseudo-clivada "torna explícita a divisão entre o dado e o novo na interação" (p. 416). Dessa forma, é possível postular que a construção pseudo-clivada funciona como uma construção operadora de intersubjetividade, na medida em que ela é utilizada em resposta (o elemento novo) a algo que foi enunciado anteriormente no fluxo discursivo (o elemento dado).

O exemplo a seguir é de uma entrevista com Cindy Sheenan, mãe de um soldado americano morto na guerra do Iraque, e que fazia na época dura oposição ao governo Bush:

(12) The rest of the world often sees the United States as a violent and dangerous country. Does Cindy Sheenan agree? Cindy Sheenan [49, a controversial figure in the US, thanks to her very personal campaign against President George W. Bush over the Iraq War]: I think that what is the most dangerous is our government...our government's trying to take our rights away from us, you know, we do have high crime rates, but the true enemy of our country right now is the Bush administration. (SPEAK UP no 238, s/d, p.15)

O tema da conversa é introduzido pelo entrevistador ao assumir a perspectiva de que os EUA são um país violento e perigoso. O que está ativado na pergunta é o modelo sócio-político de país. No entanto, a entrevistada refuta essa perspectiva ao dizer que "o que é perigoso é o governo", assumindo, assim, uma perspectiva que não se alinha com a do entrevistador, mas que perfila ou coloca em proeminência um outro elemento desse MCI de país enquanto organização sócio-política, que é o governo representado pela pessoa de George W. Bush. A construção, assim, funciona explicitamente como um recurso de intersubjetividade, 
V. 9 (2)

$214-236$

maio-ago

2019

pois emerge do fluxo discursivo e cria sentido a partir da fala do entrevistador. A CEC, reforçada pelo encaixe da construção pseudoclivada, cumpre o papel de contrastar perspectivas de forma também implícita, indicando um procedimento de disjunção cognitiva.

No exemplo a seguir, retirado do Corpus of Contemporary American English (COCA), temos novamente um frame em relevo na reportagem, retirado da revista norte americana Esquire, de 2014, em que a discussão gira em torno da tecnologia oriunda do Vale do Silício e de como eles podem beneficiar a população geral:

(13) If you want to tell me that you're optimistic about technology, what I want to know is why aren't we creating more blockbuster drugs, "Ezra Klein says. " If you want to tell me you're optimistic about technology, what I want to know is why is it still not clear how we're going to feed eleven billion people. Silicon Valley has somehow managed to brand itself as technology, but technology includes a lot of things. I think that what Silicon Valley is genuinely optimistic about is the Internet. And the problems the Internet can solve are many, but they are also limited. I think being optimistic about the world requires you to be optimistic about advances in agricultural production, advances in medicine, advances in energy. And being optimistic about how awesome the Internet is going to become is very consistent with the pessimism about the world cooking itself through the unregulated fossil-fuel emissions. "( ESQUIRE, 2014, vol. 162, nº 5, p. 128-141)

Dentro do frame de tecnologia, o entrevistado coloca em destaque um elemento específico, que é a Internet, utilizando-se, para isto, da construção pseudo-clivada, que alça esse elemento ao foco do discurso. Esta construção se coloca, sob o ponto de vista discursivo, como um contraponto à argumentação de que o Vale do Silício pode oferecer soluções tecnológicas para tudo, como a internet, porque isto não é tudo. Nesse sentido, a construção funciona como uma forma de sinalizar uma perspectiva que se contrapõe à contribuição do Vale do Silício na solução dos problemas da contemporaneidade, pois há outros aspectos inerentes à existência humana que precisam igualmente ser considerados, como a sustentabilidade na agricultura, saúde e energia. 


\section{As construções condicionais}

Quando a construção epistêmica é introduzida pelo conector de condicionalidade if, que é um operador discursivo (FAUCONNIER, 1994, 1997), duas perspectivas se apresentam, a saber: "You think that" e "You don't think that". Conforme a classificação de Sweetser (1990) para as construções condicionais, a relação causal entre prótase (p) e apódose (q) se verifica no plano epistêmico (o que ela denominou condicional epistêmica) e não no plano do conteúdo. Isso equivale a dizer que não há uma relação causal direta entre pensar o festival como algo louco e estar certo, mas "ao assumir que o ponto de vista do interlocutor/leitor é de que o festival seja algo louco, o falante/redator vai concluir que seu interlocutor/leitor pode estar certo (a esse respeito).". Tomemos o exemplo (14).

(14) Isle of Wight. If you think that a festival to celebrate a strong-smelling, pungent-tasting bulb is a crazy idea, you may be right - but that hasn't stopped the town of Newchurch from hosting their annual Isle of Wight Garlic Festival for the last 19 years. (SPEAK UP no 209, 2004, p. 6)

Argumentamos que, ao se valer da construção com o preenchimento do complementizador, o falante sinaliza implicitamente que não se alinha com a perspectiva de que o festival é algo maluco. Em termos explícitos, essa perspectiva é reforçada pela modalização do conteúdo na apódose, expressa pelo verbo modal "may": "you may be right". Em outras palavras, o falante/redator parece dizer: "eu não concordo (plenamente) com você".

Assim, a escolha da CEC, integrada à construção condicional, evidencia que a expectativa do redator não coincide com a crença (presumida) do leitor e das pessoas de forma geral, sinalizando mais uma vez um procedimento implícito de não-alinhamento de perspectivas.

No exemplo a seguir, também retirado do Corpus of Contemporary American English(COCA), temos um segundo caso de uma CEC integrada à construção condicional.

(15) If you think that presidential campaigns are all frivolous and silly and people saying stupid things, you owe an apology to Texas Governor Rick Perry. Last week 
V. 9 (2)

$214-236$

maio-ago 2019

he gave a terrific speech about how black folks need to hold Democrats accountable for their failures in terms of improving the quality of life in the black community. He says that, in fact, Republicans have lost the moral legitimacy as the party of Lincoln by not going after that black vote. Says the best social service for anybody is a job. (FOX, 2015)

No exemplo acima, novamente argumentamos que o falante se utiliza da construção condicional, que conceptualmente introduz duas perspectivas distintas no discurso: "you think that" ou "you don't think that" (all presidential campaigns are frivolous and silly). Tal construção funciona como estratégia argumentativa para apresentar a perspectiva do próprio falante: no caso específico do Governador do Texas, Rick Perry, o falante não concorda que a campanha seja "leviana e tola", já que argumenta que "devemos desculpas ao Governador do Texas", pois ele fez um "discurso incrível" (sobre o fato de os Democratas serem responsáveis pelo fracasso em melhorar as condições de vida da comunidade negra).

Argumentamos, então, que a CEC sinaliza de forma implícita o ponto de vista do falante, que se utiliza do recurso sintático explícito da construção condicional para, discursivamente, desenvolver sua argumentação a favor do discurso do governador e contrária à perspectiva de que "todas as campanhas presidenciais são levianas e tolas".

\section{As construções interrogativas}

Quando a construção epistêmica ocorre no escopo de perguntas endereçadas ao ouvinte/interlocutor/leitor, a construção com "that" é normalmente utilizada. Segundo Quirk \& Greenbaum (1973), as perguntas respondidas por "yes" e "no" "questionam a validade da predicação" (QUIRK; GREENBAUM, 1973, p. 14). A construção pode evidenciar, ainda, que o falante pressupõe a perspectiva das pessoas de forma geral, como visão socialmente compartilhada, ativando-a na sua pergunta, mas colocando-se implicitamente como não-associado a essa perspectiva.

No caso abaixo, retirado de um fórum de discussão, o tema é o questionamento sobre os garotos que gostam de jogar vídeo games, que seriam considerados nerds pelas garotas. 
(16) Do girls think that guys who play video games are nerdy? Do Girls think Gamers are Nerds? Inevitably, some do and some do not. Here in input and advice from FAQ Farmers:

a. I asked my lovely 17-year-old daughter this question. She says girls DO think that guys who play video games are nerdy, and added, "What's wrong with being a nerd, they are much better than jocks?" She does not have a lot of time for the "cool" guys. (ANSWERS, s/d)

Neste exemplo, tem-se uma CEC interrogativa que traz, na sua oração encaixada, uma informação que é atribuída a um grupo de pessoas, mas marcada implicitamente como divergente da perspectiva do entrevistador. A construção assume, dessa forma, caráter intersubjetivo.

No que diz respeito à participação das pessoas no fórum de discussão, a pergunta licencia dois tipos de resposta: "sim, os garotos são nerds" ou "não, os garotos não são nerds". A opinião de um dos participantes traz uma construção introduzida por um marcador de ênfase (do), que se alinha com uma das perspectivas ativadas na pergunta, ou seja, "os meninos são nerds". O falante reporta a opinião de sua filha e não a sua própria, que provavelmente é divergente. Esse conjunto de fatores propicia a utilização da construção complexa.

O exemplo abaixo, retirado do British National Corpus (BNC) e oriundo de uma entrevista com o artista Marcel Duchamp, gravada em 1966 e publicada no Arts Newspaper em 1993, ilustra novamente o caso da CEC integrada à construção interrogativa:

(17): The Art Newspaper: Do you think that your work would have been possible if these movements had not existed? Marcel Duchamp Absolutely not. I followed the ideas of various schools at various times, with my own reservations of course, but I was strongly influenced by each school, each time, like everyone else. No-one can escape the influences surrounding them.

The Art Newspaper: Do you think that our century will be the age of Surrealism? Marcel Duchamp: Yes, probably, but I don't know for 
V. 9 (2)

$214-236$

maio-ago

2019

sure. Deep down, I believe that our century will not be very interesting compared to other centuries. I think we will be regarded as being rather limited. Ours isn't a century like the 18th century which is impossible to love but which has its own integrity, an identity. I believe that we will be regarded as a slightly frivolous century, and that we will not be showered with the sort of praise that we have blithely been giving ourselves. (THE ART NEWSPAPER, 1993).

Neste caso, a pergunta do entrevistador se situa face aos movimentos artísticos que influenciaram o artista entrevistado, dentre eles o Dadaísmo. Ao utilizar a construção epistêmica complexa, argumentamos que o entrevistador implicitamente não se alinha com a perspectiva ativada nas perguntas, quais sejam: (i) o trabalho do artista ter sido possível sem a contribuição dos movimentos da época e (ii) o século XX ser a era do Surrealismo. Em ambas as perguntas, a construção interrogativa licencia duas possíveis respostas, positiva e negativa, que se autoexcluem. Assim, a construção interrogativa, funciona, em termos explícitos, como um recurso sintático para ativar dois possíveis pontos de vista mutuamente excludentes, ao mesmo tempo em que, integrada à CEC, implicitamente sinaliza um não alinhamento do falante com a perspectiva pressuposta na pergunta.

\section{As construções negativas}

Finalmente, outro aspecto intersubjetivo das construções completivas epistêmicas diz respeito às orações principais com verbos epistêmicos na forma negativa. Dancygier (2012), em sua pesquisa sobre negação e intersubjetividade sob o prisma da Teoria dos Espaços Mentais, argumenta que "[a construção epistêmica negativa] nega a perspectiva que o verbo expressa, e, assim, apresenta o ponto de vista do falante como negativo (em vez de positivo), em uma situação em que a perspectiva positiva poderia ser esperada ou desejada (no espaço mental alternativo)" (p.69).

Dessa forma, quando o falante tem reação negativa ao conteúdo expresso pela proposição, que Dancygier (2012) denomina espaço representacional, a construção complexa é utilizada, sinalizando uma crítica ao conteúdo da proposição e revelando, assim, que não há alinhamento com a perspectiva disponível na interação: 
(18)- Daschle: 'Certainly, I Don't Think That Many People Are Overtaxed.'

From Let Freedom Ring, by Sean Hannity, page 209:

In October of 1997, for example, then-Senate Minority Leader Tom Daschle was speaking with some journalists when he was asked if Democrats thought the American people were overtaxed, and whether he had any interest in working with Americans to cut taxes... "We have the lowest tax rate of any industrialized country in the world," Daschle replied. "That tax rate has, in large measure, been the subject of a great deal of debate about fairness for a long period of time. We have a great disparity between the richest and poorest in this country. Our view is that we've got to make the tax system more fair. But certainly I don't think that many people are overtaxed." (HANNITY, 2004)

No caso acima, o entrevistado sinaliza a necessidade de revisão do sistema de tributação no país, mas se opõe à ideia de supercobrança de impostos quando diz que "certamente não acha que muitas pessoas são sobrecarregadas com impostos". Sua perspectiva de representante dos Democratas, portanto, não se encontra em alinhamento com a que foi oferecida pelo entrevistador na pergunta.

No segundo caso de negação na oração matriz da construção epistêmica, temos:

(19) Speak Up: Dr. Tony McEnery is a linguist at Lancaster University in England. He is an expert on bad language and is currently conducting the first major study of the way in which British people swear in spoken English. I spoke to him about swearing in Britain and how it is changing. (...) From your studies, would you say that the British are a particularly foulmouthed group of people?

Tony McEnery: I don't think that the British swear any more or less than other nations or races through time. I think that, by comparison to some cultures, the taboo words and phrases in British English may be rather different. So for example, religious-based swearing in British English is very, very mild. To say something like 
V. 9 (2)

$214-236$

maio-ago

2019

'Oh, God' or 'Oh Jesus" "Jesus Christ" or "Bloody Hell" even, something like that, is incredibly mild, and I know, for other cultures, that religious swearing isn't mild at all. (SPEAK UP nº169, 2001, p. 15).

Neste exemplo, o entrevistador ativa uma determinada perspectiva na sua pergunta, ao questionar o linguista sobre o comportamento dos britânicos em termos de palavrões, inquirindo-o sobre a possibilidade de os britânicos serem "desbocados". O entrevistado responde prontamente com uma construção negativa complexa, refutando, dessa forma, a perspectiva oferecida pelo entrevistador e apresentando uma perspectiva diversa, a de que o uso desse tipo de linguagem na cultura britânica pode ser diferente, por exemplo, como no uso dos palavrões de natureza religiosa, considerado "brando" em comparação a outras culturas.

Defendemos, então, que as construções negativas sinalizam explicitamente a atenção e resposta negativa a uma perspectiva já ativada. A presença da construção com o complementizador vem sinalizar, então, de forma implícita, um processo de não-alinhamento de perspectivas, mais uma vez em um procedimento discursivo intersubjetivo de disjunção cognitiva.

\section{Considerações Finais}

O presente artigo teve por objetivo discutir o papel do conceito de (inter)subjetividade como elo construcional na rede de construções completivas, em especial em sua relação com a construção completiva epistêmica do tipo [SN VEpist [that S]], aqui denominada CEC.

Partindo de uma breve revisão da literatura contemporânea em linguística sobre os conceitos de objetividade, subjetividade e intersubjetividade, duas posturas distintas e, de certa forma, complementares foram mapeadas a respeito do conceito e de sua conceptualização no fluxo discursivo.

Compreendendo a noção como um mecanismo de natureza cognitivo-pragmático-discursiva, postulou-sequea(inter)subjetividade se verifica tanto em termos explícitos (TRAUGOTT; DASHER, 2005) quanto implícitos ao uso da língua (LANGACKER, 1987, 1990, 2008; VERHAGEN, 2005; NUYTS, 2001). 
No primeiro caso, a codificação linguística da (inter) subjetividade se deu explicitamente através de construções que funcionaram como operadores discursivos sinalizadores de atenção à perspectiva do interlocutor. No segundo caso, a codificação da intersubjetividade ocorreu de maneira implícita, na atitude do falante com relação à perspectiva ativada no fluxo discursivo, em um mecanismo de disjunção cognitiva, isto é, de não-alinhamento com perspectivas já ativadas discursivamente.

Assim, evidencia-se que estruturas sintáticas como as construções pseudo-clivadas, as construções condicionais, as construções interrogativas e as construções negativas compartilham traços de (inter)subjetividade tanto no plano linguístico quanto conceptual, possibilitando postular a noção de (inter)subjetividade como um elo conceptual que liga essas construções em rede.

\section{Referências}

ALMEIDA, Sandra. Subjetividade e intersubjetividade: as construções completivas epistêmicas em inglês. 2010. 209 f. Tese de Doutorado. Faculdade de Letras, Universidade Federal do Rio de Janeiro, Rio de Janeiro, 2010.

ALMEIDA, Sandra; FERRARI, L. V.. Subjectivity, intersubjectivity and epistemic complementation constructions. Online Proceedings of UK CLA meetings, v. 01, p. 110-127-127, 2012.

ANSWERS. S.l; s.d. Disponível em < http://wiki.answers.com>. Acesso em: 25 de julho de 2008.

CANÇADO, M. Manual de Semântica: noções básicas e exercícios. São Paulo: Contexto, 2012.

DANCYGIER, B..Negation, stance verbs and intersubjectivity. In: DANCYGIER, B.; SWEETSER, E.. Viewpoint in Language: A Multimodal Perspective. Cambridge: Cambridge University Press, 2012.

ESQUIRE. New York, vol. 162, no 5, p. 128-141, 2014. Disponível em <http:// corpus.byu.edu/bnc/>. Acesso em: 17/06/17.

FAUCONNIER, Gilles. Mental Spaces: Aspects of Meaning Construction in Natural Language. Cambridge: Cambridge University Press, 1994.

FAUCONNIER, Gilles. Mappings in Thought and Language. Cambridge: Cambridge University Press, 1997.

THE FIVE. Fox News Network. New York, 2015. Disponível em $<$ http://corpus. byu.edu/coca/>. Acesso em: 15/06/17.

GOLDBERG, A. Constructions: A Construction Grammar Approach to Argument Structure. Chicago: University of Chicago Press, 1995. 
V. 9 (2)

$214-236$ maio-ago 2019

GOLDBERG, A. Constructions at Work: The nature of Generalization in Language. New York: OUP, 2006.

HANNITY, Sean. Let Freedom Ring: Winning the War of Liberty over Liberalism. New York: Harper Collins, 2004. Disponível em < $\underline{\text { http://campaignspot.nationalreview.com/post/>. }}$. Acesso em: 28/07/09.

SPEAK UP. English Magazine. São Paulo: Peixes, 1996-2009.

LANGACKER, R. Foundations of Cognitive Grammar. v. 1: Theoretical Prerequisites. Stanford: Stanford University Press, 1987

LANGACKER, R. Subjectification. In: Cognitive Linguistics 1-1 ,5-38, 1990.

LANGACKER, R. Cognitive Grammar: A Basic Introduction. New York: Oxford University Press, 2008.

NUYTS, Jan. Subjectivity as an evidential dimension in epistemic modal expressions. Journal of Pragmatics 33, 383-400, 2001.

PALMER, F. R. Mood and Modality. Cambridge: Cambridge University Press, 1986.

QUIRK, Randolph; GREENBAUM, Sidney. A University Grammar of English. Harlow: Longman, 1973.

SWEETSER, Eve. From Etimology to Pragmatics: Metaphorical and Cultural Aspects of Semantic Structure. Cambridge: Cambridge University Press, 1990.

TOMASELLO, Michael. Origens Culturais da Aquisição do Conhecimento Humano. São Paulo: Martins Fontes, 2003.

TOMASELLO, Michael. Constructing a Language: a Usage-based Theory of Language Acquisition. Harvard: Harvard University Press, 2005.

TRAUGOTT, Elizabeth C. \& DASHER, Richard B... Regularity in Semantic Change. Cambridge: Cambridge University Press, 2005.

VERHAGEN, Arie. Constructions of Intersubjectivity: Discourse, Syntax and Cognition. New York: Oxford University Press, 2005. 